\title{
Enhanced flux pinning of YBCO/Ag composites
}

\author{
Bilal A. Malik ${ }^{1 *}$, Manzoor A. Malik ${ }^{1}$ and K. Asokan² \\ ${ }^{1}$ Department of Physics, University of Kashmir, Srinagar- 190006. \\ ${ }^{2}$ Materials Science Division, Inter University Accelerator Centre, New Delhi -110067. \\ *Email: bilal200620@gmail.com
}

Soon after the discovery of high temperature superconductivity, it was realized the practical application in these materials is restricted by weak flux pinning and complex vortex dynamics [1-3]. Since high temperature superconductors (HTSCs) have short coherence length of several nanometers it provides the opportunity to restrict the motion of flux lines by introducing secondary phase over the dimensions of coherence length [4-6] that results enhancement of superconducting properties. In this study, we have introduced silver $(\mathrm{Ag})$ particles in $\mathrm{YBa}_{2} \mathrm{Cu}_{3} \mathrm{O}_{7}$ (YBCO) over the dimension of coherence length. Due to the metallic nature of silver, it apart from acting as strong pinning centers also increases grain coupling thus avoids its brittleness. The effects were probed in terms of magnetization under applied magnetic field up to 14 $\mathrm{T}$ by vibrating sample magnetometer and results are shown in Figure 1(a). The critical current density is calculated using Beans model [7] and is plotted in Fig 1(b). Fig 2 (a) shows the variation of critical current density $\left(\mathrm{J}_{\mathrm{C}}\right)$ with the addition of $\mathrm{Ag}$ in YBCO and the variation of pinning force $\left(J_{C} \times H\right)$ with magnetic field for different composites is shown in Fig 2(b) It was found that critical current density increases six times and pining force also increases ten times by the addition of $15 \% \mathrm{Ag}$ in pure YBCO thereby making YBCO more attractive material for technolgical application. In conclusion we found addition of Ag particles of several nanomters in YBCO acts as strong artifical pinning centres even at high magnetic fields.

\section{Refrences}

1. A.C. Wright, K. Zhang, A. Erbil, Phys. Rev. B 44 (1991) 2 .

2. G. Blatter et al. Rev. Mod. Phys. 66(1994) 1125

3. V.M. Galitski, G. Refael, M.P.A. Fisher, T. Senthil, Phys. Rev. Lett. 95 (2005) 077002.

4. T. Haugan, P.N. Barnes, R. Wheeler, F. Meisenkothen, M. Sumption, Nature 430 (2004) 867.
5. M.N. Hasan, M. Kiuchi, E.S. Otabe, T. Matsuhita, M. Muralidhar, Supercond. Sci. Technol. 20 (2007) 345 .

6. A. Kujur, D. Behera, Journal of Magnetism and Magnetic Materials 377 (2015) 34.

7. C. P. Bean, Phys. Rev. Lett. 8 (1962) 250
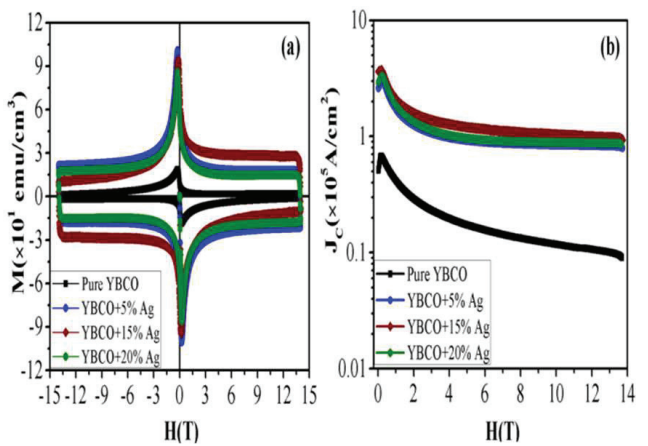

Figure 1: (a) Isothermal Magnetization hysteresis loops of $\mathrm{YBCO}+\mathrm{xAg}(\mathrm{x}=0,5,15$, and 20 wt. \%) composites samples at $5 \mathrm{~K}(\mathrm{~b})$ variation of $J_{C}$ as a function of applied magnetic field
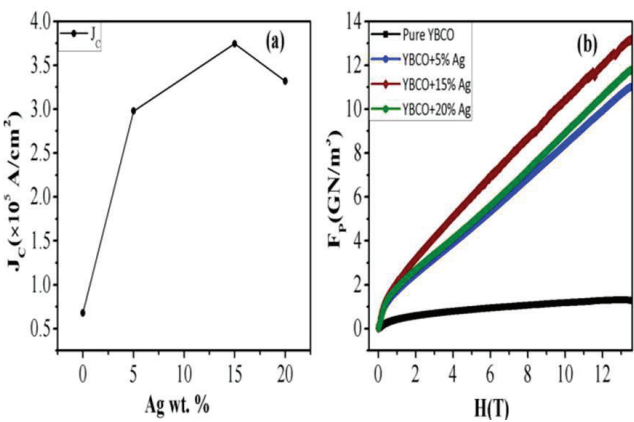

Figure 2: (a) Variation of critical current density with BZO wt.\% (b) Pinning force density of YBCO+ $\mathrm{xAg}(\mathrm{x}=0,5,15$, and $20 \mathrm{wt}$. \%) composites samples as a function of applied magnetic field. 